\title{
ANÁLISE DO GOOGLE SITES COMO UMA PLATAFORMA OPEN PARA A GESTÃO DE PROJETOS DE EQUIPES DE ESTUDANTES DE ENGENHARIA
}

Cláudia Andressa Cruz Affonso (claudia.andressa@ifsp.edu.br) - Instituto Federal de Educação, Ciência e Tecnologia de São Paulo

Daniel Capaldo Amaral (amaral@sc.usp.br) - Universidade de São Paulo

\section{RESUMO}

O Processo de Desenvolvimento de Produtos (PDP) por meio de colaboração aberta em comunidades de projetistas e usuários, em um regime copyleft e rede peer to peer virtual caracteriza o fenômeno denominado Open Source Design (OSD). As comunidades que aderem a este fenômeno disponibilizam um conjunto de informações capazes de permitir a fabricação remota em que o usuário pode construir o seu produto, ao invés de um sistema produtivo específico previamente definido, como no desenvolvimento tradicional. A tangibilidade e a distância física entre desenvolvedores impõem barreiras à colaboração aberta e peer to peer, mas avanços como a tecnologia de impressão $3 D$ fez surgir plataformas virtuais, mais especificamente, plataformas open (abertas), para o compartilhamento de arquivos tornando esta realidade possivel. O presente estudo analisou a plataforma Google Sites como um meio para introduzir os conceitos de OSD na aprendizagem baseada em problemas (Problem-Based Learning - PBL) em equipes de estudantes de engenharia. $O$ objetivo consistiu em identificar e analisar as propriedades da plataforma Google Sites consideradas pelas equipes de projetos como críticas para o sucesso de um gerenciamento de projetos em uma plataforma aberta. O estudo foi conduzido em equipes de estudantes de Engenharia de Produção em uma disciplina de PDP. A percepção dos integrantes das equipes foi mensurada por meio de um questionário aplicado individualmente no qual se analisou as propriedades da plataforma previstas pela teoria. As respostas individuais dos membros das seis equipes foram analisadas por meio do indice de concordância e foi realizada uma verificação se as propriedades identificadas entre os estudantes se aproximavam daquelas previstas em plataformas open. Entre as propriedades, o índice de concordância foi forte para: a apresentação do projeto e versionamento. Enquanto a padronização das informações, estrutura do produto, papéis de competências, gerenciador de arquivos, imprecisão no trabalho cooperativo e ambiente controlado tiveram concordância moderada.

Palavras-chave: Colaboração; Plataforma; Open Source Design; Desenvolvimento de Produtos; Problem-Based Learning

Área: Educação para desenvolvimento de produtos e serviços

\section{INTRODUÇÃO}

As organizações evoluíram ao longo dos anos envolvendo os consumidores em atividades específicas no processo de design, especialmente aquelas de testes de escolhas nas fases finais do PDP, como agente passivo desse processo (ATKINSON, 2006). Um envolvimento maior é dificultado por barreiras como a falta de capacitação e linguagem técnica, necessárias para interagir com os projetistas nas fases iniciais. Os avanços tecnológicos em ferramentas digitais, realizados nas últimas décadas, estão tornando possível a criação de interface amigáveis e mais próximas do usuário não especialista, ou sem formação específica em Desenvolvimento de Produtos (DP). Isso pode significar novas formas de produção e de design, em que os produtos sejam criados com a participação ainda mais intensa e por meio de modelos de negócio que envolvam a participação de leigos ou usuários comuns (ILAN, 2011). 
O conceito de Open Source Design (OSD) está associado a este movimento. Ele utiliza princípios similares aos de indivíduos das comunidades Open Source Software, em que o software é criado e refinado por voluntários. O movimento do software livre vislumbrou a formação de uma nova modalidade de organização produtiva, radicalmente descentralizada, colaborativa e sem "proprietário", baseada em recursos compartilhados e resultados altamente distribuídos, conecta indivíduos irrestritamente os quais cooperam uns com os outros sem preocupar-se com o mercado ou comandos gerenciais (BENKLER, 2006, p.60). Embora as comunidades de usuários sejam mais conhecidas para o desenvolvimento de software, como por exemplo, Linux, Apache e Firefox, elas não estão mais restritas a este tipo de produto (JONG; BRUIJN, 2013). Já existem comunidades como RepRap, Open Source Ecology, Enable, Velocar e Open Source Vehicle que estão buscando o desenvolvimento compartilhado e livre e voltadas para produtos manufaturados.

Müller-Seitz e Reger (2010) destacam um dos principais obstáculos do projeto OScar (Open Source Car) - um projeto desenvolvido em 1999 com o objetivo de desenvolver um carro de forma aberta e livre, por meio da internet, sem restrições e direitos autorias, sem chefia e sem hierarquia. O obstáculo consistia na dificuldade de troca de componentes (peças) do carro via internet. O que no OSS era resolvido de forma simples, visto que o código-fonte ou uma versão compilada de um software transita facilmente na internet ou outros meios digitais. Outro estudo realizado por Affonso e Amaral (2015a) na comunidade Open Source Ecology mostrou que a revisão e os testes dos componentes das máquinas desenvolvidas são fracos, dada a dificuldade da replicabilidade e do alto custo de um protótipo completo do produto.

Recentemente, porém, novos avanços estão alterando o quadro. A tecnologia de impressão 3D amadureceu e está se tornando um diferencial diminuindo esta barreira. Em tese, hoje ela realiza a ponte entre objetos virtuais e objetos para o mundo real permitindo aos projetistas diversas interações, tendo amostras físicas para avaliar cada conceito (DIMITROV; SCHREVE; BEER , 2006). Esses avanços da tecnologia de impressão 3D (BANKS, 2013; BEM; PUPO; PEREIRA, 2013) resultaram no surgimento de outro facilitador para o desenvolvimento OSD, as plataformas virtuais para o compartilhamento de arquivos para impressão 3D, e, ao seu redor, profissionais e usuários que se propõem a desenvolver produtos neste novo modelo organizacional colaborativo de desenvolvimento aberto.

Uma análise de casos OSD realizada por Affonso e Amaral (2015b) indicou que a maior parte do desenvolvimento aberto nestas comunidades ocorria por meio de discussões em plataformas como o Google+ e para o compartilhamento das atividades desenvolvidas, cada membro utiliza um (ou mais) repositório (s) que mais lhe convém, tais como: Dropbox, Thingiverse, Youtube, Instructables, entre outros. Com base nisso, o presente estudo analisou a plataforma Google Sites como um meio para introduzir os conceitos de OSD na aprendizagem baseada em problemas (Problem-Based Learning - PBL) em equipes de estudantes de engenharia. O objetivo consiste em identificar e analisar as propriedades da plataforma Google Sites consideradas pelas equipes de projetos como críticas para o sucesso de um gerenciamento de projetos em uma plataforma aberta.

Com intuito de auxiliar no gerenciamento das inúmeras informações geradas pelos projetistas em plataformas abertas, foi realizado um estudo relatando a experiência das equipes de projetos no desenvolvimento de produtos utilizando o método PBL. Estudo conduzido em equipes de estudantes de Engenharia de Produção em uma disciplina de PDP. O desafio consistiu em gerenciar o projeto por meio de um site criado pelas equipes de estudantes na plataforma Google Sites. O site da equipe atuava como uma plataforma para criar, compartilhar e contribuir com os conteúdos hospedados. Neste artigo o estudo foi estruturado nas seções: (i) revisão de literatura, descrevendo os conceitos de plataformas abertas e 
aprendizagem baseada em problemas (PBL); (ii) método de pesquisa; (iii) Propriedades críticas da plataforma Google Sites; e, por fim, (iv) considerações finais.

\section{REVISÃO DA LITERATURA}

\subsection{Plataformas open}

A estratégia colaborativa e aberta de desenvolvimento de produtos manufaturados em que os participantes estão interligados em uma rede peer to peer é definida aqui como Open Source Design. A efetivação desta estratégia ocorre por meio de plataformas open as quais estabelecem a mediação entre os artefatos compartilhados e os membros de um mesmo projeto de maneira distribuída. Essas plataformas necessariamente se hospedam no espaço virtual da internet. Todos os membros destas plataformas utilizam algum tipo de meio digital para compartilhamento de artefatos e disponibilização do resultado do desenvolvimento. A literatura apresenta diversas definições sobre os meios utilizados para disponibilizar os variados tipos de artefatos.

Em desenvolvimento de produtos, o banco de dados denominado data vault prevê o armazenamento e recuperação de informações do produto (LIU; XU, 2001). Pinfield (2014, p.165), por sua vez, faz uso do termo repositório para este meio digital, definido por como "um conjunto de sistemas e serviços que facilitam a inserção, o armazenamento, gerenciamento, recuperação, exibição e reuso dos objetos digitais." Szykman (2000) acrescenta que um repositório de design é um sistema de modelagem de artefato de design baseado no conhecimento, utilizado para facilitar a representação, a captura, compartilhamento e reutilização do conhecimento de design corporativo. O conceito de plataforma é utilizado pelos autores West (2002), Boudreau (2010) e Fjeldsted et al (2012). E está associado a habilitar, facilitar e empoderar as interações e desenvolvimento entre os participantes por meio de uma relação simbiótica (FJELDSTED et al., 2012).

Este artigo utilizará o termo plataforma open, dado que no contexto OSD em que as atividades são livres de direitos autorais, a plataforma não atua apenas como uma base de armazenamento de artefatos, mas também como um meio de interação entre os participantes dispersos globalmente, que participarão de um ou mais processos de desenvolvimento de produtos manufaturados e, portanto, necessitam de um ambiente aberto para alcançarem resultados efetivos.

Plataforma open é definida como: uma interface virtual que favorece o desenvolvimento colaborativo de produtos manufaturados, por meio da criação, armazenamento, compartilhamento, gerenciamento, exibição e reuso de objetos digitais (PINFIELD, 2014, p.165). O objetivo consiste em habilitar, facilitar e dar autonomia para a colaboração em projetos open source (FJELDSTED et al, 2012).

\subsection{Aprendizagem baseada em problemas (PBL)}

A aprendizagem baseada em problemas é uma abordagem instrucional centrada no aprendizado a qual capacita o aprendiz a conduzir pesquisa, integrar teoria com prática e aplicar conhecimento e habilidades de desenvolvimento para uma solução viável para um problema definido (SAVERY, 2015).

Hansen (2006) destaca que a PBL usa problemas complexos e reais para motivar os alunos a identificar e pesquisar os conceitos e princípios que precisam saber para resolver esses problemas. Os objetivos do PBL são ajudar os alunos:

a) a pensar de forma crítica, analisar e resolver problemas complexos do mundo real; 
b) encontrar, avaliar e usar recursos de aprendizado;

c) trabalhar cooperativamente em equipe;

d) demonstrar habilidades de comunicação efetivas; e,

e) usar conhecimento de conteúdo e habilidades intelectuais para se tornar aprendentes contínuos (HANSEN, 2006).

\section{MÉTODO DE PESQUISA}

O estudo foi realizado em uma turma de 44 alunos do curso de Engenharia de Produção enquanto cursavam a disciplina de Processo de Desenvolvimento do Produto oferecida no $8^{0}$ semestre da grade curricular. Os estudantes foram agrupados em seis equipes, havendo quatro equipes com sete estudantes e duas com oito estudantes. Dois grandes desafios foram apresentados aos estudantes:

- O primeiro consistiu em desenvolver um utensílio para pia de cozinha, com base nas fases inicias do PDP de Rozenfeld et al. (2006). Os problemas relativos às fases do modelo de PDP do autor mencionado eram quinzenalmente dados aos estudantes. As fases apresentadas que compuseram os problemas foram: Planejamento estratégico do produto, Planejamento do projeto, Projeto Informacional e Projeto Conceitual;

- O segundo desafio fundamentava-se na criação de um site para o gerenciamento do projeto utilizando a plataforma Google Sites. Para tanto, o professor e a monitora da disciplina atuaram como facilitadores neste processo de criação do site. O objetivo deste desafio não se restringia apenas na criação do site, mas na utilização dele como um meio para compartilhamento e contribuição dos integrantes das equipes nos conteúdos produzidos. A escolha do Google Sites justifica-se pela facilidade de criação e manutenção das páginas da web, além da rapidez dado que a velocidade do Google Sites se apoia nos servidores do Google. Não obstante, a aplicação estruturada de wikis e criação de páginas na web permite aos envolvidos uma criação dinâmica de páginas web (LIN et al., 2014).

- Baseado em Yew; Schmidt (2012), uma breve descrição das fases do processo de PBL apresentado quinzenalmente aos estudantes pode ser observada nas cinco fases:

- Fase 1: Apresentação do problema (aproximadamente $30 \mathrm{~min}$ ) - o professor apresentava o problema e exemplos de práticas que poderiam ser empregadas nos projetos das equipes;

- Fase 2: Análise do problema (aproximadamente $30 \mathrm{~min}$ ) - as equipes se reuniam para identificar os problemas mais pertinentes ao seus projetos e se organizavam para a busca do conhecimento prévio necessário;

- Fase 3: Aprendizagem autodirigida (aproximadamente 1h30min) - as equipes se organizavam para realização das pesquisas individuais. Parte dos estudantes buscava conteúdo online na internet, um no máximo dois integrantes cuidavam da criação do site e o restante dos integrantes trabalhava em planilhas, organização de pesquisas de mercado e tabulação dos dados;

- Fase 4: Reunião da equipe com o professor e monitor (aproximadamente $30 \mathrm{~min}$ ) Cada equipe se reúne com o professor e o monitor para compartilhar seu progresso, relatar suas dúvidas e estratégia de compreensão do problema. 
- Fase 5: Consolidação e relatório (1h) - as equipes consolidam seus estudos em relatório, descrevendo a resposta ao problema identificado

Ao final do semestre ocorreu uma última fase com o propósito de mostrar a síntese do projeto. Nesta fase os alunos apresentam dois artefatos: slides do Microsoft Power Point todo o estudo realizado para o desenvolvimento do utensílio de cozinha, apresentação essa feita pela equipe com duração de aproximadamente 30 minutos; e disponibilizam o site desenvolvido na plataforma Google Sites. A plataforma Google sites foi escolhida como o meio investigado para o gerenciamento dos projetos desenvolvidos pelos alunos em uma disciplina de PDP do curso de Engenharia de Produção. Para análise deste último artefato foi elaborado e aplicado um questionário com o objetivo de analisar quais seriam as propriedades críticas de sucesso da plataforma de acordo com a percepção dos estudantes.

A teoria de plataformas open evidencia propriedades críticas a este meio. Em sua Revisão Bibliográfica Sistemática (RBS) Affonso e Amaral (2015b) evidenciaram muitas destas propriedades, as quais são resultantes dos trabalhos de Star (2010), Carlile (2002) e Galdzicki et al. (2014). Este artigo utiliza como variável de estudo essas apontadas na RBS acrescentada de outras publicadas um pouco mais recentemente por Bonvoisin e Boujut (2015). As propriedades são:

- apresentação do projeto, rede social, papéis competências/ habilidades, ferramentas de design para suporte, expressão dos requisitos, compilação do conhecimento, versionamento, comunicação interpessoal síncrona e assíncrona, espaço de comunicação aberta/natureza iterativa (BONVOISIN e BOUJUT, 2015);

- padronização das informações/formato formalizado, tarefas/atividades padronizadas, gerenciador de arquivos/criação de projetos hierárquicos e complexidade reduzida (GALDZICKI et al., 2014);

- estrutura modular, imprecisão no trabalho cooperativo, ambiente controlado e heterogeneidade interna (STAR, 2010)

- glossário/abordagem sintática (CARLILE, 2002 e GALDZICKI et al., 2014).

No total foram 18 propriedades analisadas por meio de 19 afirmações, conforme Apêndice A. Essas foram apresentadas em um questionário aplicado individualmente para cada membro das equipes e cada afirmação possuía uma escala de 1 (discordo plenamente) a 10 (concordo plenamente). $\mathrm{O}$ índice de concordância foi a técnica estatística empregada para analisar os dados coletados. Este índice analisou o julgamento de cada estudante e a concordância entre os vários membros da mesma equipe em relação a uma determinada afirmação. $O$ índice de concordância foi calculado de acordo com a Equação 1 por Lebreton e Senter (2008).

$$
\mathrm{r}_{\mathrm{wg}}=1-\left(\mathrm{S}_{\mathrm{X}}^{2} / \sigma_{\mathrm{E}}^{2}\right)
$$

Em que $S^{2}$ representa a variância observada de $X ;$ e, $\sigma_{E}^{2}$ corresponde a variância esperada quando há completa falta de concordância entre os participantes.

O valor de corte utilizado, tradicionalmente, para um alto nível de concordância é de 0,7 (LEBRETON; SENTER, 2008). O software utilizado para o tratamento dos resultados obtidos foi SPSS - o PASW Statistics 17.0.

\section{PROPRIEDADES CRÍTICAS DA PLATAFORMA GOOGLE SITES}

Após o desenvolvimento de todas as fases do PBL descritas no método, foi aplicado aos estudantes um questionário com as afirmações indicadas no Apêndice A. O objetivo foi 
analisar a percepção dos estudantes em relação às propriedades que poderiam ser críticas para o gerenciamento de projeto na plataforma Google Sites. Dois exemplos de sites criados pelas equipes podem ser visualizados nas Figuras 1 e 2.

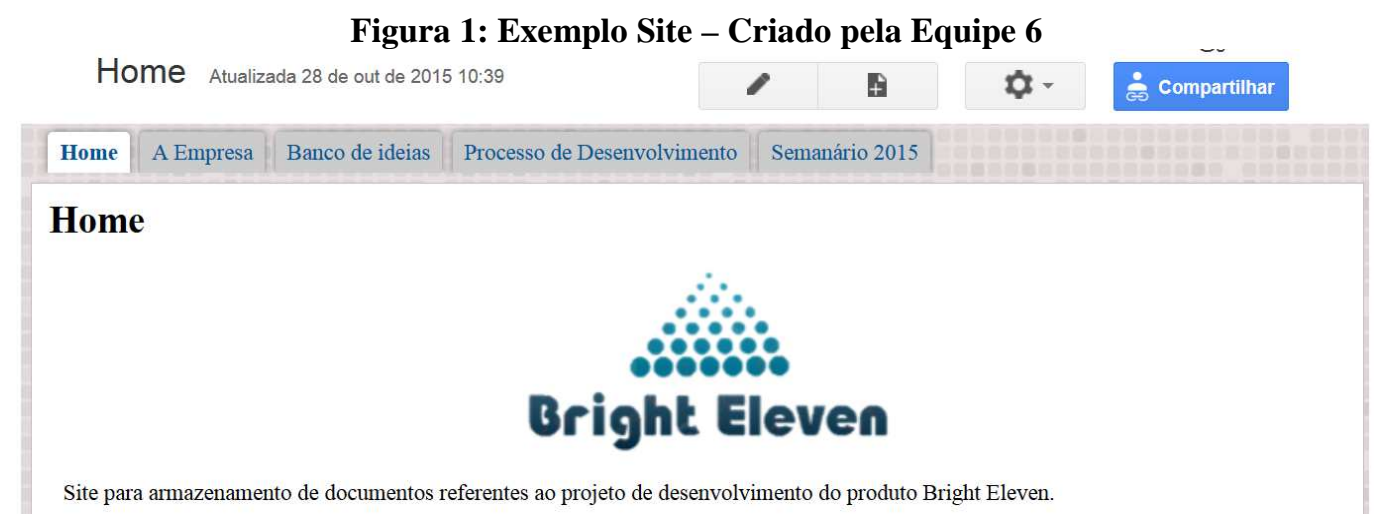

Fonte: Adaptado Grupo Bright Eleven (estudantes da disciplina PDP)

Pode-se observar que cada equipe teve autonomia para criar a interface do seu site, conforme as suas necessidades. O exemplo da Figura 1 mostrou um site que a tela inicial possuía poucas informações sobre o projeto a ser desenvolvido. A equipe que desenvolveu o site apresentado na Figura 2, por sua vez, optou por um site mais direto sobre as necessidades e entregas do projeto, inserindo inclusive um gadget com calendário para atualização das necessidades.

Figura 2: Exemplo Site - Criado pela Equipe 2

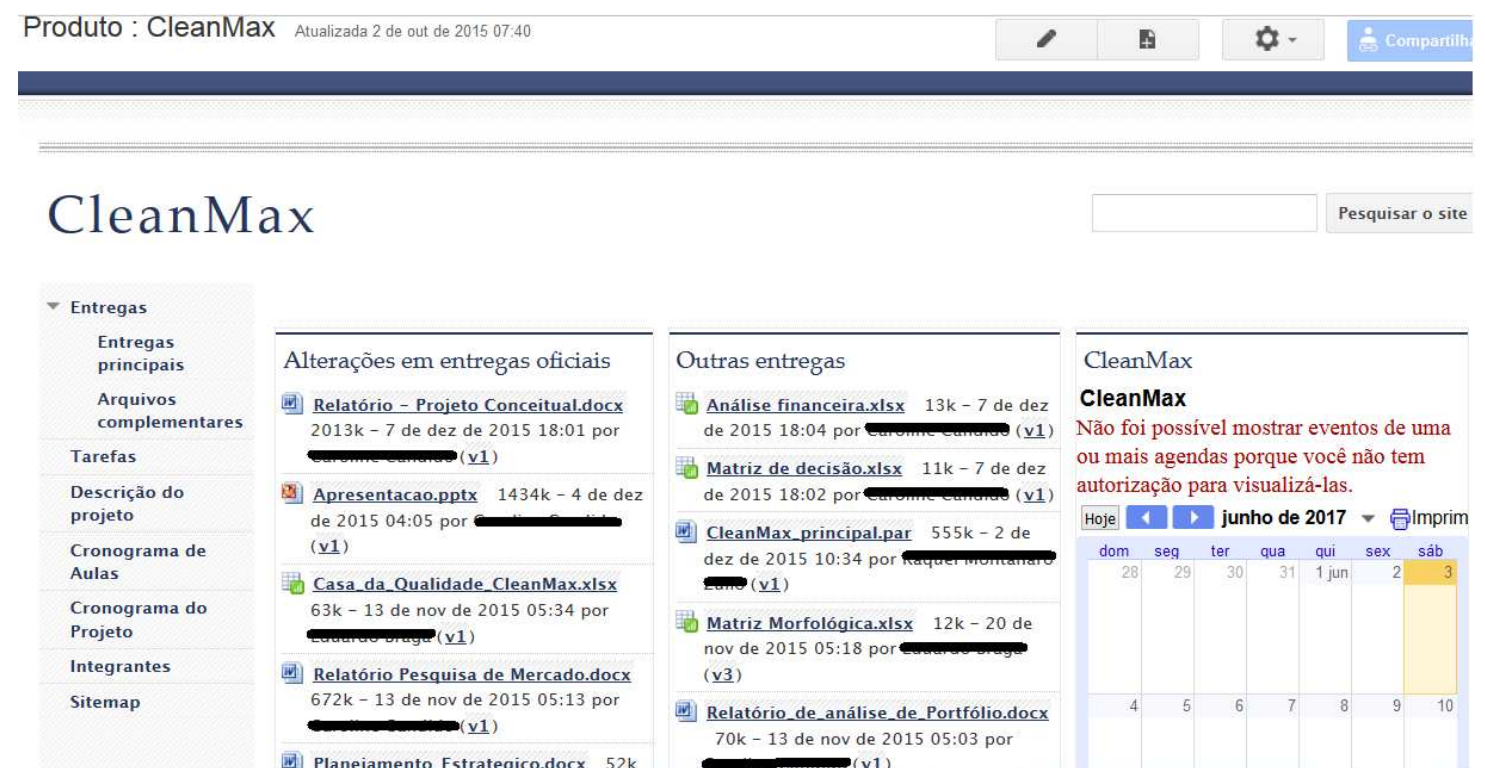

Fonte:Adaptado de Grupo CleanMax (estudantes da disciplina PDP)

Após a criação dos sites, aplicou-se um questionário aos estudantes. Entre os 44 estudantes das seis equipes foram obtidos 30 questionários válidos para análise, conforme Tabela 1. 
Tabela 1: Caracterização do total da amostra

\begin{tabular}{lcr}
\hline Equipe & $\begin{array}{c}\text { Número de } \\
\text { questionários válidos }\end{array}$ & $\begin{array}{c}\text { \% de questionários válidos em } \\
\text { relação ao total da amostra }\end{array}$ \\
1 & 3 & $43 \%$ \\
2 & 3 & $43 \%$ \\
3 & 4 & $57 \%$ \\
4 & 5 & $71 \%$ \\
5 & 7 & $87,5 \%$ \\
6 & 8 & $100 \%$ \\
\hline
\end{tabular}

Fonte - Elaboração própria

Os resultados mostraram que as propriedades indicadas como críticas na plataforma Google Sites e com $r_{\mathrm{wg}} \geq 0,7$ foram: a apresentação do projeto e versionamento. Enquanto a padronização das informações, estrutura do produto, papéis e competências, gerenciador de arquivos, imprecisão no trabalho cooperativo e ambiente controlado tiveram concordância moderada $\left(0,50 \leq \mathrm{r}_{\mathrm{wg}}<0,70\right)$.

A primeira propriedade (apresentação do projeto) consiste na definição de todas as informações relativas ao projeto. Neste sentido a fase 2 do PBL quando realizada auxiliou na definição destes parâmetros pela equipe. A segunda propriedade (versionamento) aponta a necessidade de controle de versão de documentos na plataforma. $\mathrm{O}$ objetivo é não haver retrabalho e que se torne rotina o uso da versão mais recente disponibilizada pela equipe.

A propriedade 3 (padronização das informações) remete a dificuldade em encontrar documentos que foram disponibilizados pela equipe na plataforma. Isso ocorria devido a não padronização entre os membros sobre como as informações seriam disponibilizadas. Os integrantes relataram a necessidade de uma sistemática para uma linguagem mais universal entre eles. A quarta propriedade (estrutura do produto) consiste na necessidade de criar uma estrutura para o DP na plataforma Google Sites que mostre de forma concisa a modularidade do produto que está sendo construído. A necessidade desta modularidade só foi percebida pelos estudantes nas fases finais do projeto. A propriedade 5 (papéis e competências) indica a facilidade no cumprimento das atividades por parte dos estudantes quando havia uma definição clara dos papéis que cada um desempenharia. A sexta propriedade (gerenciador de arquivos) descreve a necessidade de uma hierarquização dos documentos dos projetos, dado que foi relevante o número de estudantes que relataram a replicação de documentos, o mesmo material sendo produzido por mais de um individuo sem que houvesse a necessidade.

A sétima propriedade (imprecisão no trabalho) relata a necessidade de autonomia requisitada pelos integrantes da equipe, a plataforma deve permitir a colaboração sem impor muitos padrões. Portanto, embora sistemáticas sejam necessárias para controle e compartilhamento de documentos, como apontados nas propriedades 2, 3 e 6, o grau destas sistemáticas deve ser moderado para que não se engesse o processo, tornando a colaboração uma atividade desgastante. E por fim, a última propriedade (ambiente controlado) evidencia a necessidade de um moderador (governança) para controlar os comentários e debates na equipe.

\section{CONSIDERAÇÕES FINAIS}

A abordagem PBL auxiliou no trabalho em equipes dos estudantes para adquirir, comunicar e integrar informações (HANSEN, 2006). Neste sentido, a plataforma atuou como um meio 
diferenciado nesta abordagem. Entender as propriedades que viabilizam a colaboração aberta e peer to peer, compõem um dos objetivos deste estudo. Para tanto, a principal contribuição desta pesquisa consistiu na análise da percepção dos estudantes quanto a plataforma Google Sites como um meio para esse tipo de desenvolvimento.

O versionamento e a apresentação dos parâmetros do projeto foram apontados como essenciais ao PDP nas plataformas. E outro fator de destaque foram as propriedades que tiveram um índice de concordância moderado. Embora, a imprecisão do trabalho seja apontada como um aspecto relevante neste tipo de colaboração, a necessidade de um procedimento e/ou algum tipo padrão para tornar mais universal a colaboração é exigida. Observa-se, dessa forma, um trade-off que merece uma investigação, em que é preciso algum grau de sistematização sem que haja um engessamento para a colaboração nas plataformas.

Apesar de ter sido um primeiro esforço, o tamanho da amostra investigada foi muito reduzido, o que não possibilita uma generalização dos resultados encontrados, de qualquer forma foi possível observar os benefícios e a concordância entre os indivíduos quanto às propriedades investigadas. Sugere-se como trabalhos futuros: a aplicação deste estudo em uma amostra maior; uma análise da influência dessas essas propriedades no contexto OSD; e, um estudo sobre o trade-off identificado.

\section{REFERÊNCIAS}

AFFONSO, C. A. C.; AMARAL, D. C. Boundary objects in Open Source Design: experiences from OSE community. International Conference on Engineering Design 2015, ICED 2015. Milan, Italy, 2015a.

AFFONSO, C. A. C.; AMARAL, D. C. Caracterização de repositórios como Boundary Objects para comunidades Open Source Design. In: Congresso Brasileiro de Gestão da Inovação e Desenvolvimento de Produtos, 2015, Itajubá. $10^{\circ} \mathrm{CBGDP}$ (Congresso Brasileiro de Gestão da Inovação e Desenvolvimento de Produtos), 2015 b.

ATKINSON, P.. Do it yourself: Democracy and design. Journal of Design History, v.19, n.1, p.1-10, 2006.

BANKS, J. Adding value in additive manufacturing: Researchers in the United Kingdom and Europe look to 3D printing for customization. IEEE Pulse, v. 4, n.6, p. 22-26, 2013

BEM, G. M., PUPO, R. T., PEREIRA, A. T. C. . Additive manufacturing as a social inclusion tool. In Green Design, Materials and Manufacturing Processes - Proceedings of the 2nd International Conference on Sustainable Intelligent Manufacturing, SIM , p.63-66, 2013.

BENKLER, Y. The wealth of networks: how social production transforms markets and freedom. Yale University Press: New Haven and London, 2006.

BONVOISIN, J., BOUJUT, J. F. Open design platforms for open source product development: current state and requirements. In Innovation and Creativity, Proceedings of the 20th International Conference on Engineering Design,v. 8, p. 11-22, 2015.

BOUDREAU, K. Open platform strategies and innovation: Granting access vs. devolving control. Management Science, v. 56, n. 10, p. 1849-1872, 2010.

CARLILE, P.R.A Pragmatic View of Knowledge and Boundaries. Organization Science, 2002.

DIMITROV , D., SCHREVE , K., BEER , N. DE. Advances in three dimensional printing state of the art and future perspectives. Rapid Prototyping Journal, 2006. 
FJELDSTED, A.et al. Open source development of tangible products. In: DS 71: Proceedings of NordDesign 2012, the 9th NordDesign conference, Aarlborg University, Denmark. 2224.08. 2012. 2012.

GALDZICKI, M.et al. The Synthetic Biology Open Language (SBOL) provides a community standard for communicating designs in synthetic biology. Nature biotechnology, v. 32, n. 6, p. 545-550, 2014.

HANSEN, J. D. Using problem-based learning in accounting. Journal of Education for Business, v. 81, n. 4, p. 221-224, 2006.

ILAN, Z. B. From Economy of Commodities to Economy of Ideas: Hardware as Social Medium. Design Management Review. v.22, n. 3, p.: 44-53, 2011.

JONG, J.; BRUIJN, E. Innovation Lessons From 3-D Printing. MIT Sloan Management Review, v.54, n.2, p.43-52, 2013.

LIN, Yen-Ting et al. A cloud-based learning environment for developing student reflection abilities. Computers in Human Behavior, v. 32, p. 244-252, 2014.

LIU, D. T; XU, X. W. A review of web-based product data management systems. Computers in industry, v. 44, n. 3, p. 251-262, 2001.

MÜLLER-SEITZ, G., REGER, G. Networking beyond the software code? an explorative examination of the development of an open source car project. Technovation, v. 30, n. 11-12, p. 627-634, 2010.

PINFIELD, S.et al. Open-access repositories worldwide, 2005-2012: Past growth, current characteristics, and future possibilities. Journal of the association for information science and technology, v. 65, n. 12, p. 2404-2421, 2014.

ROZENFELD, H. et al. Gestão de Desenvolvimento de Produtos: uma referência para a melhoria do processo. São Paulo: Saraiva, 2006. 542 p.

SAVERY, J. R. Overview of problem-based learning: Definitions and distinctions. Essential readings in problem-based learning: Exploring and extending the legacy of Howard S. Barrows, p. 5-15, 2015.

STAR, S. L.. This is not a boundary object: Reflections on the origin of a concept. Science, Technology, \& Human Values, v. 35, n. 5, p. 601-617, 2010.

SZYKMAN, S. et al. Design repositories: engineering design's new knowledge base. IEEE Intelligent Systems and Their Applications, v. 15, n. 3, p. 48-55, 2000.

WEST, J. How open is open enough? Melding proprietary and open source platform strategies. Research policy, v. 32, n. 7, p. 1259-1285, 2003.

YEW, Elaine HJ; SCHMIDT, Henk G. What students learn in problem-based learning: A process analysis. Instructional Science, v. 40, n. 2, p. 371-395, 2012. 


\section{APÊNDICE A - PROPRIEDADES E AFIRMAÇÕES PARA ANÁLISE DA PLATAFORMA GOOGLE SITES}

\begin{tabular}{|c|c|}
\hline Propriedade & Afirmação \\
\hline Apresentação do projeto & $\begin{array}{l}\text { Acredito que a definição do que seria o projeto } \\
\text { (incluindo informações como: objetivos, missão, valores, } \\
\text { visão) no site da equipe ajudou no alcance do resultado } \\
\text { final }\end{array}$ \\
\hline Rede social & $\begin{array}{l}\text { Acredito que se o site criado pela equipe funcionasse } \\
\text { como uma rede social em que pessoas de todo o } \\
\text { mundo pudessem contribuir o desenvolvimento do } \\
\text { produto seria melhor }\end{array}$ \\
\hline $\begin{array}{l}\text { Padronização das } \\
\text { informações/Formato } \\
\text { padronizado }\end{array}$ & $\begin{array}{l}\text { Tive problemas para encontrar/buscar algum documento } \\
\text { disponibilizado pelo grupo }\end{array}$ \\
\hline Estrutura de produto modular & $\begin{array}{l}\text { Acredito que a organização das informações no google } \\
\text { sites seria mais eficiente se ela tivesse sido organizada de } \\
\text { acordo com a estrutura do produto (BOM- bill of } \\
\text { materials) }\end{array}$ \\
\hline $\begin{array}{l}\text { Tarefas/Atividades } \\
\text { padronizadas }\end{array}$ & $\begin{array}{l}\text { Senti falta de uma instrução previamente estabelecida } \\
\text { pela equipe para realizar as atividades que me foram } \\
\text { atribuidas }\end{array}$ \\
\hline $\begin{array}{l}\text { Papéis e } \\
\text { competências/habilidades }\end{array}$ & $\begin{array}{l}\text { Tive facilidade em cumprir as atividades, já que possuia } \\
\text { mais competências e habilidades no que me foi atribuido }\end{array}$ \\
\hline $\begin{array}{l}\text { Ferramentas de design para } \\
\text { suporte }\end{array}$ & $\begin{array}{l}\text { Precisei buscar outras ferramentas de design free } \\
\text { (ex.sketchup) para apoio ao processo de } \\
\text { desenvolvimento do produto }\end{array}$ \\
\hline Expressão dos requisitos & $\begin{array}{l}\text { Consegui expressar/desdobrar/trabalhar facilmente em } \\
\text { todos os requisitos do produto }\end{array}$ \\
\hline Compilação do conhecimento & $\begin{array}{l}\text { Foi necessário um fechamento (sintese) final dos } \\
\text { documentos para reduzir o excesso de redundância e } \\
\text { informações que não agregavam ao projeto }\end{array}$ \\
\hline $\begin{array}{l}\text { Gerenciador de } \\
\text { arquivos/Criação de projetos } \\
\text { hierárquicos }\end{array}$ & $\begin{array}{l}\text { Tivemos replicação de documentos, ou seja, o mesmo } \\
\text { documento feito por mais de uma pessoa sem } \\
\text { necessidade }\end{array}$ \\
\hline \multirow[t]{2}{*}{ Versionamento } & $\begin{array}{l}\text { Verificava frequentemente as contribuições que os } \\
\text { outros integrantes faziam a partir de documentos já } \\
\text { criados, isto é, verificava as versões mais novas }\end{array}$ \\
\hline & $\begin{array}{l}\text { Usava a última versão do documento/relatório para } \\
\text { seguir com minhas contribuições }\end{array}$ \\
\hline $\begin{array}{l}\text { Comunicação interpessoal } \\
\text { sincrona }\end{array}$ & $\begin{array}{l}\text { Utilizei Hangouts ou/e outro mecanismo para } \\
\text { comunicação simultânea via repositório pelo menos uma } \\
\text { vez }\end{array}$ \\
\hline $\begin{array}{l}\text { Comunicação interpessoal } \\
\text { assincrona }\end{array}$ & $\begin{array}{l}\text { Utilizei posts/fóruns existentes no google sites para } \\
\text { comunicação entre os integrantes da equipes }\end{array}$ \\
\hline $\begin{array}{l}\text { Espaço de comunicação } \\
\text { aberta/natureza iterativa }\end{array}$ & $\begin{array}{l}\text { Participei via Google Sites de discussões aberta sobre o } \\
\text { projeto }\end{array}$ \\
\hline Complexidade reduzida & Achei fácil a utilização do Google Sites \\
\hline $\begin{array}{l}\text { Imprecisão no trabalho } \\
\text { cooperativo }\end{array}$ & $\begin{array}{l}\text { Tinha autonomia em colaborar da forma que julgasse } \\
\text { mais adequada, sem seguir padrões pré-estabelecidos }\end{array}$ \\
\hline $\begin{array}{l}\text { Ambiente controlado e } \\
\text { heterogeneidade interna }\end{array}$ & $\begin{array}{l}\text { Senti falta de um responsável(is) especifico(s) da equipe } \\
\text { para controlar os comentários e debates sobre o projeto }\end{array}$ \\
\hline $\begin{array}{l}\text { Glossário e abordagem } \\
\text { sintática e representação }\end{array}$ & $\begin{array}{l}\text { Senti falta de algo como um glossário para entender } \\
\text { determinados termos utilizados pelos membros da } \\
\text { equipe durante o processo de desenvolvimento de } \\
\text { produto }\end{array}$ \\
\hline
\end{tabular}

